\title{
Prevalence of metallo- $\beta$-lactamase genes among Pseudomonas aeruginosa isolated from various clinical samples in China
}

https://doi.org/10.1515/labmed-2019-0162

Received September 19, 2019; accepted January 29, 2020

\section{Abstract}

Background: Pseudomonas aeruginosa is an opportunistic pathogen which is associated with nosocomial infections and causes various diseases including urinary tract infection, pneumonia, soft-tissue infection and sepsis. The emergence of $P$. aeruginosa-acquired metallo- $\beta$ lactamase (MBL) is most worrisome and poses a serious threat during treatment and infection control. The objective of this study was to identify antibiotic susceptibility, phenotypic detection of MBL production and to determine the prevalence of MBL genes in carbapenem-resistant $P$. aeruginosa isolated from different clinical samples.

Methods: A total of 329 non-duplicate $P$. aeruginosa isolated from various clinical samples from two hospitals in China between September 2017 and March 2019 were included in this study. Phenotypic detection of MBL was performed by the combined detection method using imipenem and imipenem-ethylenediaminetetraacetic acid (EDTA) discs. MBL-encoding genes including bla ${ }_{V I M-1}$,

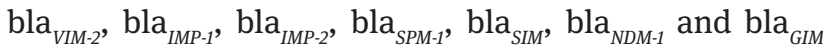
were detected by polymerase chain reaction (PCR).

Results: Of the 329 P. aeruginosa, majority of the isolates were resistant to imipenem $(77.5 \%)$ followed by meropenem $(64.7 \%)$. Of the $270 \mathrm{P}$. aeruginosa isolates tested, 149 (55.2\%) isolates were found to be positive for MBL detection. Of the different samples, 57.8\% $(\mathrm{n}=26)$ of $P$. aeruginosa isolated from blood were found to be positive for MBL production. Of the various MBL genes, bla ${ }_{I M P-1}$ (28.2\%) was the most predominant gene detected followed by bla $\mathrm{VIM-2}(18.8 \%)$, bla ${ }_{V I M-1}(16.1 \%)$, bla ${ }_{N D M-1}(9.4 \%)$,

\footnotetext{
*Correspondence: Wei Wang, Department of Laboratory Medicine, Beijing Friendship Hospital, Capital Medical University, Beijing 100050, P.R. China, Phone/Fax: 0086-010-63138541, E-Mail:wangwei_112233@163.com Xiaoya Wang: Department of Neurosurgery, Nanchong Central Hospital, The Second Clinical Medical College, North Sichuan Medical College, Nanchon, Sichuan, P.R. China
}

Ә Open Access. (C) 2020 Wei Wang et al., published by De Gruyter. (c) BY Public License.

Published online July 16, 2020
$\operatorname{bla}_{I M P-2}(6.7 \%), \operatorname{bla}_{S I M}(6.0 \%)$, bla $_{S P M-1}(4.0 \%)$ and bla $_{G I M}$ (1.3\%) genes.

Conclusions: The high resistance of $P$. aeruginosa toward imipenem and meropenem and the high prevalence of bla $_{I M P-1}$ and bla ${ }_{V I M-2}$ set the alarm on the increasing, perhaps the increased, carbapenem resistance. In addition to routine antibiotic susceptibility testings, our results emphasize the importance of both the phenotypic and genotypic MBL detection methods in routine practice for early detection of carbapenem resistance and to prevent further dissemination of this resistant pathogen.

Keywords: carbapenem resistance; metallo- $\beta$-lactamase genes; Pseudomonas aeruginosa.

\section{Introduction}

Pseudomonas aeruginosa, an opportunistic pathogen, is associated with nosocomial infections and causes various diseases including pneumonia, urinary tract infection, soft-tissue infection and sepsis [1]. Infections caused by multidrug-resistant (MDR) P. aeruginosa are associated with significant morbidity and mortality. A high level of intrinsic and acquired resistance to multiple antibiotics exhibited by $P$. aeruginos $a$ makes it challenging to treat and limits the treatment options [2]. Pseudomonas aeruginosa exhibits almost all known resistance mechanisms; however, enzyme production is the major mechanism of acquired resistance, especially $\beta$-lactamase production [3]. The increased prevalence of extended-spectrum $\beta$-lactamase (ESBL)-producing $P$. aeruginosa led to the use of carbapenems. Carbapenems are the last choice of drug for the treatment of $P$. aeruginosa infections. However, the alarming increase in carbapenem resistance is a cause of serious concern in the treatment of $P$. aeruginosa infections [4]. The World Health Organization (WHO) has identified 12 most common bacteria that pose a challenge to human health. Among these, carbapenem-resistant $P$. aeruginosa was designated as one of the highly critical and poses a serious threat to patients who require ventilators and 
blood catheters [5]. $\beta$-lactamases are classified into four different classes: classes $\mathrm{A}, \mathrm{C}$ and $\mathrm{D}$ act through a serinebased mechanism and metallo- $\beta$-lactamase (MBL), while class $B$ requires a bivalent metal ion for its activity [6]. Of all these mechanisms, the emergence of $P$. aeruginos $a$ acquired MBL is the most worrisome and poses a serious threat during treatment and infection control [7]. Except for monobactams, MBL-producing strains can hydrolyze all other $\beta$-lactam antibiotics including penicillin, cephamycins, cephalosporin and carbapenems [8]. The MBLproducing $P$. aeruginos $a$ strains carry co-resistance genes for other classes of antibiotics.

The MBL genes are present in mobile genetic elements such as plasmids, transposons, integrons or associated with insertion sequences with a tendency to spread within species and between different species [8]. Several types of MBL genes were identified in P. aeruginosa: (i) Verona integron-encoded metallo- $\beta$-lactamase (VIM), (ii) imipenemase (IMP), Seoul imipenemase (SIM), (iii) Germany imipenemase (GIM), (iv) São Paulo metallo- $\beta$-lactamase (SPM), (v) New Delhi metallo- $\beta$-lactamase (NDM) types. Of these, VIM and IMP are the most prevalent types of acquired MBLs [9, 10]. In 1991, MBL resistance was first reported in Japan; later it was reported in various countries including China, India, Taiwan, Singapore, Korea, Italy, France, Greece, Australia, Germany, Austria, Turkey, Bulgaria, Netherlands, Spain, Mexico, Colombia and USA [11]. Mortality due to MBL-producing $P$. aeruginosa ranged from $70 \%$ to $90 \%$ [11, 12].

When patients with severe infections caused by MBLproducing $P$. aeruginosa are treated with antibiotics, it often leads to poor clinical outcome. Thus, it is highly essential to detect MBL-producing P. aeruginosa as early as possible for the effective treatment of critically ill patients within clinical settings. The present study aimed to identify antibiotic susceptibility, phenotypic detection of MBL and to determine the prevalence of MBL genes in carbapenem-resistant $P$. aeruginos $a$ isolated from different clinical samples.

\section{Materials and methods}

\section{Bacterial strains}

A total of 329 non-duplicate $P$. aeruginosa isolated from various clinical samples from the Beijing Friendship Hospital, Capital Medical University, Beijing, China and The Second Affiliated Hospital of North Sichuan Medical College, Nanchong, Sichuan, China between September
2017 and March 2019 were included in this study. The isolates were identified as P. aeruginosa using the VITEK 2 system (bioMérieux, Craponne, France). Demographic data and other relevant details of patients from whom $P$. aeruginosa was isolated were collected from the hospital medical record department. The Institutional Ethical Board approved the study (IRB: 037-2017).

\section{Susceptibility testing}

Antibiotic susceptibility test for all P. aeruginosa isolates was performed by disc diffusion method on Mueller-Hinton agar (MHA) plates. The following antibiotics were used during the test: imipenem $(10 \mu \mathrm{g})$, meropenem $(10 \mu \mathrm{g})$, gentamicin $(10 \mu \mathrm{g})$, amikacin $(30 \mu \mathrm{g})$, cefotaxime (30 $\mu \mathrm{g})$, ceftazidime $(30 \mu \mathrm{g})$, norfloxacin $(10 \mu \mathrm{g})$, ciprofloxacin $(5 \mu \mathrm{g})$, piperacillin + tazobactam $(100 / 10 \mu \mathrm{g})$, colistin $(10 \mu \mathrm{g})$ and tigecycline $(15 \mu \mathrm{g})$ (Himedia, Mumbai, India). The results were interpreted as per the Clinical Laboratory Standard Institute (CLSI) guidelines [13].

\section{Phenotypic detection of MBL production}

All isolates that were resistant and intermediately resistant to imipenem by disc diffusion method were subjected to MBL production test by the combined disk method [14]. Briefly, overnight culture adjusted to 0.5 MacFarland standard was inoculated on to MHA plates. Two disks of 10 $\mu \mathrm{g}$ imipenem $(10 \mu \mathrm{g})$ and imipenem $(10 \mu \mathrm{g})$ with $0.5 \mathrm{M}$ ethylenediaminetetraacetic acid (EDTA) were placed $25 \mathrm{~mm}$ apart on the MHA plates. The plates were incubated at 37 ${ }^{\circ} \mathrm{C}$ for $16-18 \mathrm{~h}$ and observed for the zone of inhibition. A zone of $\geq 7 \mathrm{~mm}$ in the imipenem plus EDTA disc compared to imipenem alone disc was considered as positive for the presence of carbapenem resistance (MBL resistance).

\section{Detection of MBL genes}

Carbapenem-resistant isolates were subjected to polymerase chain reaction (PCR) for the detection of MBL genes

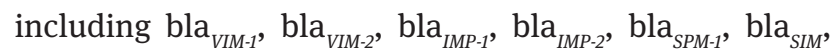
bla $_{N D M-1}$ and bla ${ }_{G I M}$ as described by Azimi et al. [15]. Inhouse $P$. aeruginosa isolates positive for all tested genes and confirmed earlier through sequencing were used as controls for PCR. The gene-specific primer sequence and their annealing temperature are presented in Table 1 [16-18]. DNA was extracted using a commercial genomic DNA extraction kit (Thermo Fisher Scientific, Waltham, 
Table 1: Gene-specific primer sequence.

\begin{tabular}{|c|c|c|}
\hline Gene & Primer sequence $\left(5^{\prime}-3^{\prime}\right)$ & $\begin{array}{r}\text { Annealing } \\
\text { temperature, }{ }^{\circ} \mathrm{C}\end{array}$ \\
\hline \multirow[t]{2}{*}{ bla $_{V I M-1}$} & F: 5'-AGTGGTGAGTATCCGACAG-3' & 53 \\
\hline & R: 5'-ATGAAAGTGCGTGGAGAC-3' & \\
\hline \multirow[t]{2}{*}{ bla $_{V I M-2}$} & F: 5'-ATGTTCAAACTTTTGAGTAAG-3' & 52 \\
\hline & R: 5'-CTACTCAACGACTGAGCG-3' & \\
\hline \multirow[t]{2}{*}{ bla $_{\text {IMP-1 }}$} & F: $5^{\prime}$-ACCGCAGCAGAGTCTTTGCC-3' & 55 \\
\hline & R: 5'-ACAACCAGTTTTGCCTTACC-3' & \\
\hline \multirow[t]{2}{*}{ bla $_{\text {IMP-2 }}$} & F: 5'-GTTTTATGTGTATGCTTCC-3' & 51 \\
\hline & R: 5'-AGCCTGTTCCCATGTAC-3' & \\
\hline \multirow[t]{2}{*}{ bla $_{S P M-1}$} & F: 5'-GCGTTTTGTTTGTTGCTC-3' & 53 \\
\hline & R: 5'-TTGGGGATGTGAGACTAC-3' & \\
\hline \multirow{2}{*}{ bla $_{S I M}$} & F: 5'-TACAAGGGATTCGGCATCC-3' & 52 \\
\hline & R: 5'-TAATGGCCTGTTCCCATG-3' & \\
\hline \multirow[t]{2}{*}{$\mathrm{bla}_{N D M-1}$} & F: 5'-GGCGGAATGGCTCATCACGA-3' & 56 \\
\hline & R: 5'-CGCAACACAGCCTGACTTTC- $3^{\prime}$ & \\
\hline \multirow[t]{2}{*}{ bla $_{G I M}$} & F: 5'-TCGACACACCTTGGTCTG-3' & 52 \\
\hline & R: 5'-AACTTCCAACTTTGCCAT-3' & \\
\hline
\end{tabular}

MA, USA). The PCR reaction mixture contained $12.5 \mu \mathrm{L}$ of ReadyMix ${ }^{\mathrm{TM}}$ Taq PCR Reaction Mix (Sigma-Aldrich, St. Louis, MO, USA), $2.5 \mu \mathrm{L}$ of the DNA (20 pg), and $0.5 \mu \mathrm{M}$ of each primer and nuclease-free water made up to $25 \mu \mathrm{L}$. The PCR cycling conditions were as follows: initial denaturation at $96{ }^{\circ} \mathrm{C}$ for $10 \mathrm{~min}$, followed by 30 cycles of $96{ }^{\circ} \mathrm{C}$ for $1 \mathrm{~min}$, the specific annealing temperatures of the respective primers as presented in Table 1 for $1 \mathrm{~min}$, and $72{ }^{\circ} \mathrm{C}$ for $1 \mathrm{~min}$, and a final extension step at $72{ }^{\circ} \mathrm{C}$ for 10 min. After PCR, the amplicons were resolved in $1.2 \%$ $(w / v)$ agarose gel electrophoresis and visualized under a ultraviolet (UV) transilluminator (BioRad, Hercules, CA, USA).

\section{Statistical analysis}

Descriptive statistics were performed to determine the frequencies. The chi-square $\left(\chi^{2}\right)$ test, Student's t-test and Pearson's correlation coefficient tests were performed to analyze the data using SPSS statistical software (IBM SPSS Inc., Chicago, IL, USA, Ver. 2015). A p-value of $<0.05$ was considered statistically significant.

\section{Results}

\section{Patient characteristics}

A total of 329 P. aeruginosa were isolated from 329 nonrepetitive clinical samples collected from 307 patients (mean age, $49.7 \pm 6.7$ years); of these, $171(55.7 \%)$ were male and 136 (44.3\%) were female. Various clinical samples included sputum ( $n=92,28.0 \%)$, burnt wounds $(n=71$, 21.6\%), bronchoalveolar lavage (BAL) $(n=63,19.1 \%)$, blood $(n=45,13.7 \%)$, pus $(n=33,10.0 \%)$ and others $(n=25,7.6 \%)$. Of the 307 patients, $171(55.7 \%)$ patients were admitted in the intensive care unit (ICU) for various critical disease conditions (Table 2). Of the 307 patients, in 10 patients, two different samples were collected and in six patients, three different samples were collected.

\section{Susceptibility testing}

Of the 329 P. aeruginosa, majority of the isolates were resistant to imipenem $(255,77.5 \%)$ followed by meropenem (213, 64.7\%), amikacin (209, 63.5\%), gentamicin (201, $61.1 \%)$, norfloxacin $(187,56.8 \%)$, piperacillin/tazobactam $(185,56.2 \%)$, cefotaxime $(184,55.9 \%)$, ciprofloxacin $(175$, $53.2 \%)$, tigecycline $(172,52.3 \%)$, ceftazidime $(167,50.8 \%)$ and colistin (53,16.1\%). Compared to other samples, a higher number of $P$. aeruginosa $(71,77.2 \%)$ isolated from sputum samples were found to be resistant to imipenem $(p>0.05)$. All isolates that were resistant to meropenem were found to be resistant to imipenem. The correlation

Table 2: Patient demographics and clinical condition.

\begin{tabular}{lr}
\hline Description & $\begin{array}{r}\text { No. of patients/ } \\
\text { samples, } \%\end{array}$ \\
\hline Patients $(\mathrm{n}=307)$ & \\
Male & $171(55.7 \%)$ \\
Female & $136(44.3 \%)$ \\
Age (mean \pm SD) & $49.7 \pm 6.7$ years \\
In ICU & $171(55.7 \%)$ \\
In ward & $136(44.3 \%)$ \\
Under ventilator support & $89(28.3 \%)$ \\
On catheter & $56(18.2 \%)$ \\
Prior antibiotic therapy & $237(77.2 \%)$ \\
Clinical condition & \\
Respiratory disorder & $127(41.4 \%)$ \\
Burnt wound & $80(26.1 \%)$ \\
Septicemia & $72(23.5 \%)$ \\
Diabetes & $19(6.2 \%)$ \\
Urinary tract infection & $9(2.9 \%)$ \\
Clinical samples ( $=329)$ & \\
Sputum & $92(28.0 \%)$ \\
Burnt wound & $71(21.6 \%)$ \\
Bronchoalveolar lavage & $63(19.1 \%)$ \\
Blood & $45(13.7 \%)$ \\
Pus & $33(10.0 \%)$ \\
Urine & $25(7.6 \%)$ \\
\hline U &
\end{tabular}

ICU, intensive care unit; SD, standard deviation. 
coefficient showed that there was a significant association between the $P$. aeruginos $a$ isolated from patients with respiratory diseases and imipenem resistance $(r=0.98$, $\mathrm{p}=0.029)$. Thirty-two $(9.7 \%)$ isolates were found to be susceptible to all the antibiotics tested. A total of 207 (62.9\%) isolates were found to be MDR strains as they were intermediately or fully resistant to at least three different classes of antibiotics. None of the $P$. aeruginosa isolated from blood samples was resistant to colistin (Table 3).

\section{Phenotypic detection of $M B L$ production}

All the isolates that were resistant $(255,77.5 \%)$ and intermediately resistant $(15,4.6 \%)$ to imipenem were subjected to MBL detection by the combined disk method. Of the 270 P. aeruginosa isolates tested, 149 (55.2\%) isolates were found to be positive for MBL detection. Of the different samples, 57.8\% (26/45) of $P$. aeruginosa isolated

Table 3: Antibiotic susceptibility testing.

\begin{tabular}{lrrr}
\hline Antibiotics & Susceptible & Intermediate & Resistant \\
\hline Imipenem & $59(17.9 \%)$ & $15(4.6 \%)$ & $255(77.5 \%)$ \\
Meropenem & $84(25.5 \%)$ & $32(9.7 \%)$ & $213(64.7 \%)$ \\
Amikacin & $72(21.9 \%)$ & $48(14.6 \%)$ & $209(63.5 \%)$ \\
Gentamicin & $94(28.6 \%)$ & $34(10.3 \%)$ & $201(61.1 \%)$ \\
Norfloxacin & $121(36.8 \%)$ & $21(6.4 \%)$ & $187(56.8 \%)$ \\
Piperacillin/ & $117(35.6 \%)$ & $27(8.2 \%)$ & $185(56.2 \%)$ \\
tazobactum & & & \\
Cefotaxime & $91(27.1 \%)$ & $54(16.4 \%)$ & $184(55.9 \%)$ \\
Ciprofloxacin & $122(37.1 \%)$ & $32(9.7 \%)$ & $175(53.2 \%)$ \\
Tigecycline & $119(36.2 \%)$ & $38(11.6 \%)$ & $172(52.3 \%)$ \\
Ceftazidime & $114(34.7 \%)$ & $48(14.6 \%)$ & $167(50.8 \%)$ \\
Colistin & $227(69.0 \%)$ & $53(14.9 \%)$ & $53(16.1 \%)$ \\
\hline
\end{tabular}

from blood were found to be positive for MBL, followed by $P$. aeruginosa isolated from pus $(18 / 33,54.5 \%)$, sputum (41/92, 44.6\%), burnt wound (31/71, 43.7\%), BAL (24/63, $38.1 \%)$ and urine $(9 / 25,36.0 \%)$. The MBL was predominantly detected in $P$. aeruginosa isolated from male (83, $55.7 \%)$ compared to female $(66,44.3 \%)$ patients (Table 4). The presence of MBL among $P$. aeruginosa isolated from different samples did not differ significantly $(\mathrm{p}>0.05)$.

\section{Detection of MBL genes}

All the 149 P. aeruginosa that were positive for carbapenem resistance were subjected to MBL gene detection by PCR. Of the various MBL genes tested, bla $_{I M P-1}(41,28.2 \%)$ was the most common gene detected from the isolates followed by the bla VIM-2 $_{2}(28,18.8 \%)$, bla VIM-1 $_{1}(24,16.1 \%)$, bla ${ }_{N D M-1}$ (14, 9.4\%), bla $_{I M P-2}(10,6.7 \%)$, bla $_{S I M}(9,6.0 \%)$, bla $_{S P M-1}(6$, $4.0 \%)$ and $\mathrm{bla}_{G I M}(2,1.3 \%)$ genes. The presence of the bla $_{I M P-1}$ gene was significantly higher among the isolates tested $(\mathrm{p}=0.023)$. Of the various combinations identified, the presence of the bla ${ }_{I M P-1}$ gene along with $b a_{I M P-2}$ was the most common combination of genes present among the isolates (Table 5). Fourteen (9.4\%) isolates did not amplify any of the genes tested.

\section{Discussion}

Pseudomonas aeruginosa infections are effectively treated by carbapenems; however, resistance toward these antibiotics spread across hospitals due to the extensive use of antibiotics [19]. Intrinsic resistance among $P$. aeruginosa

Table 4: Phenotypic detection of MBL-producing P. aeruginosa.

\begin{tabular}{|c|c|c|c|c|c|c|}
\hline Antibiotics & $\begin{array}{l}\text { Sputum } \\
(n=92)\end{array}$ & $\begin{array}{r}\text { Burnt wound } \\
\qquad(n=71)\end{array}$ & $\begin{array}{r}\text { BAL } \\
(n=63)\end{array}$ & $\begin{array}{r}\text { Blood } \\
(n=45)\end{array}$ & $\begin{array}{r}\text { Pus } \\
(n=33)\end{array}$ & $\begin{array}{r}\text { Urine } \\
(n=25)\end{array}$ \\
\hline Imipenem & 86 (93.5\%) & $57(80.3 \%)$ & 45 (71.4\%) & $29(64.4 \%)$ & $21(63.6 \%)$ & 17 (68.0\%) \\
\hline Meropenem & $75(81.5)$ & 65 (91.5\%) & $33(52.4 \%)$ & $20(44.4 \%)$ & $12(36.4 \%)$ & $8(32.0 \%)$ \\
\hline Amikacin & 41 (44.6\%) & $52(73.2 \%)$ & $51(80.9 \%)$ & $22(48.9 \%)$ & $24(72.7 \%)$ & 19 (76.0\%) \\
\hline Gentamicin & 42 (45.7\%) & $51(71.8 \%)$ & 47 (74.6\%) & $21(46.7 \%)$ & $26(78.8 \%)$ & $14(56.0 \%)$ \\
\hline Norfloxacin & 56 (60.9\%) & $41(57.7 \%)$ & $26(41.3 \%)$ & $28(62.2 \%)$ & $21(63.6 \%)$ & $15(60.0 \%)$ \\
\hline $\begin{array}{l}\text { Piperacillin/ } \\
\text { tazobactum }\end{array}$ & $48(52.2 \%)$ & 61 (85.9\%) & 24 (38.1\%) & $21(46.7 \%)$ & 17 (51.5\%) & $14(56.0 \%)$ \\
\hline Cefotaxime & $54(58.7 \%)$ & 40 (56.3\%) & $32(50.8 \%)$ & $23(51.1 \%)$ & $23(69.7 \%)$ & $12(48.0 \%)$ \\
\hline Ciprofloxacin & $42(45.7 \%)$ & $53(74.6 \%)$ & $38(60.3 \%)$ & 19 (42.2\%) & 14 (42.4\%) & $9(36.0 \%)$ \\
\hline Tigecycline & 36 (39.1\%) & $41(57.7 \%)$ & $44(69.8 \%)$ & $18(40.0 \%)$ & $17(51.5 \%)$ & $16(64.0 \%)$ \\
\hline Ceftazidime & 71 (77.2\%) & $36(50.7 \%)$ & 21 (33.3\%) & 22 (48.9\%) & $9(27.3 \%)$ & $8(32.0 \%)$ \\
\hline Colistin & 18 (19.6\%) & $15(21.1 \%)$ & 6 (9.5\%) & 0 (0.0\%) & $9(27.3 \%)$ & $5(20.0 \%)$ \\
\hline
\end{tabular}

BAL, bronchoalveolar lavage; MBL, metallo- $\beta$-lactamase. 
Table 5: Distribution of MBL genes among $P$. aeruginosa.

\begin{tabular}{lr}
\hline Gene combination & $\begin{array}{r}\text { No. of } \\
\text { isolates }\end{array}$ \\
\hline bla $_{I M P-1}$ & $41(27.5 \%)$ \\
bla $_{V I M-2}$ & $28(18.8 \%)$ \\
bla $_{V I M-1}$ & $24(16.1 \%)$ \\
bla $_{N D M-1}$ & $14(9.4 \%)$ \\
bla $_{I M P-2}$ & $10(6.7 \%)$ \\
bla $_{\text {SIM }}$ & $9(6 \%)$ \\
bla $_{S P M-1}$ & $6(4 \%)$ \\
bla $_{G I M}$ & $2(1.3 \%)$ \\
bla $_{I M P-1}$, bla $_{I M P-2}$ & $6(4.0 \%)$ \\
bla $_{V I M-1}$, bla $_{V I M-2}$ & $4(2.7 \%)$ \\
bla $_{I M P-1}$, bla $_{N D M-1}$ & $4(2.7 \%)$ \\
bla $_{S I M}$, bla $_{I M P-2}$ & $2(1.3 \%)$ \\
bla $_{N D M-1}$, bla $_{V I M-1}$, bla $_{I M P-1}$ & $2(1.3 \%)$ \\
bla $_{N D M-1}$, bla $_{G I M}$, bla $_{I M P-1}$ & $2(1.3 \%)$ \\
bla $_{N D M-1}$, bla $_{V I M-1}$, bla $_{I M P-1}$, bla $_{I M P-2}$ & $1(0.7 \%)$ \\
\hline
\end{tabular}

MBL, metallo- $\beta$-lactamase.

leads to MDR and is associated with high mortality. This study reported a high level of carbapenem resistance among $P$. aeruginosa isolated from various clinical samples. Although not significant, P. aeruginosa (77.2\%) isolated from sputum samples were found to be resistant to imipenem $(p>0.05)$. The majority of our isolates were from sputum samples $(28.0 \%)$ and the majority of our sputum samples (72.4\%) were from patients with respiratory diseases. A significant association between the $P$. aeruginosa isolated from patients with respiratory diseases and imipenem resistance $(r=0.98, p=0.029)$ was found in this study. In our hospital, imipenem is one of the most commonly used antibiotics as first-line therapy for most of the cases with pseudomonas infection, especially for respiratory diseases and patients who are on ventilator support. An overexposure of this drug could possibly be the reason for this higher rate of imipenem resistance among our isolates.

A wide range of resistant mechanisms make P. aeruginos $a$ swiftly change to the selective environmental pressure and makes it resistant to several classes of antibiotics. In this study, $77.5 \%$ of the isolates were found to be resistant to imipenem, which corroborates to that reported from Egypt (78.3\%) [20] and China (73.3\%) [21]. Compared to other studies from the Asia-Pacific region including Iran $(25.2 \%)$ [22], China (17.1\%, 43.3\%) [23, 24], Japan (28.5\%) [25] and Taiwan (16\%) [26], this study reported a much higher resistance toward imipenem. Another study from Iran reported a resistance of $98.8 \%$ [15]. In a review article, Hong et al. presented an overview of the epidemiology and molecular characteristics of MBL-producing P. aeruginosa.
The study which analyzed several publications from Asia, Europe, America and Africa reported imipenem resistance among $P$. aeruginosa ranging from $8 \%$ to $66 \%$ [9]. In this study, meropenem resistance was the second most common resistance reported (64.7\%) among our isolates. In addition to imipenem resistance, Hong et al. reported meropenem resistance ranging from $8 \%$ to $57 \%$ [9]. In the aforementioned study, which covers multiple countries of all regions, the reported imipenem and meropenem resistance ranges among $P$. aeruginos a do not exceed the rate reported in this study [9]. This implies that imipenem and meropenem resistance among our isolates was higher than that reported worldwide and needs special attention. In addition to the overuse of imipenem and meropenem in our hospital settings, the presence of resistant genes on mobile genetic elements may have also contributed to the spread of resistance within our isolates [8]. In our study, $62.9 \%$ of the isolates were found to be MDR strains, which was higher than that reported from Brazil (37\%) and Asia $(42.8 \%)[27,28]$.

In this study, $55.2 \%$ of the isolates were found to be MBL producers. A study from Iran, which included $P$. aeruginosa isolated from burnt wound, reported a lower rate $(43.7 \%)$ of MBL producers compared to this study [15]. Similarly, another study from Iran also reported a lower rate $(37.7 \%)$ of MBL producers among their clinical isolates of $P$. aeruginosa [29]. Hong et al. in a review article analyzed reports with varied carbapenem resistance rates. Compared to this study, the review article reported lower carbapenem-resistant isolates from South and Southeast Asia including Philippines (31.1\%), Singapore (23.3\%), Thailand (28.7\%), Vietnam (46.7\%) and India (32\%); Oceanic including Australia (16\%) and New Zealand (10.3\%); East Africa including Kenya (13.7\%); and North America including Canada (3.3\%) and the United States (20\%). A much higher rate was reported from Russia (75.3\%) and Costa Rica (63.1\%) [9]. A meta-analysis from Iran, which included 14 publications on $P$. aeruginosa isolated from burn patients, reported a pooled prevalence rate of $76.8 \%$ carbapenemresistant $P$. aeruginosa, which is much higher than that reported in this study [30]. However, a study from China, which included $P$. aeruginosa isolated from cystic fibrosis patients, reported that $56.25 \%$ of their isolates were found to be MBL producers, which is comparable to that reported in this study [21]. Although not significant, a relatively high number $(41,27.52 \%)$ of P. aeruginosa isolated from sputum samples were found to be MBL producers. However, in terms of percentage, $P$. aeruginosa isolated from blood samples yielded a higher rate (57.8\%) of MBL producers. Pseudomonas aeruginosa isolated from male 
(55.7\%) patients were found to be the predominant MBL producers.

Most of the MBL genes are located in the integrons within mobile genetic elements and are responsible for the dissemination of antibiotic resistance through horizontal gene transfer. In the past decade, the emergence and dissemination of the newly identified bla $\mathrm{NDM}_{\mathrm{NM}}$ and $\mathrm{bla}_{\mathrm{AIM}}$ and the most prevalent bla $_{I M P}$, bla $_{V I M}$, bla ${ }_{S I M}$ and $b a_{G I M}$ MBL genes have been widely reported around the world [20]. In this study, bla $_{I M P-1}(28.2 \%)$ was the most common gene detected; bla VIM-2 $(18.8 \%)$ was the second most common gene detected, followed by bla VIM-1 $_{1}(16.1 \%)$. Similar to this study, a study from Iran which included MBL-producing $P$. aeruginosa reported that bla $_{I M P}(24.65 \%)$ was the predomi-

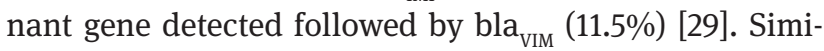
larly, a study from Japan which included 180 P. aeruginosa reported that bla $_{\text {IMP. } 1}$ was the predominant gene detected (116/180 isolates) followed by bla $_{\text {VIM-2 }}$ (63/180 isolates) [16]. While a meta-analysis from Iran which included $P$. aeruginosa from burn patients reported that the pooled prevalence of $\mathrm{bla}_{\mathrm{VIM}}$ was $21.4 \%$ and of bla $_{I M P}$ was $13.1 \%$, the reported rates were higher than in this study [30]. A study from Iran also reported that bla $\mathrm{vIM}_{\mathrm{I}}(17.5 \%)$ was the most common gene followed by bla ${ }_{I M P}(15.6 \%)$ [15]. A study from China that included $63 \mathrm{MBL}$-producing $P$. aeruginosa reported that $\mathrm{bla}_{\mathrm{vIM}}$ was the most common gene but with a higher rate $(84.1 \%)$ and $b_{I M P}$ was the second most common gene $(76.1 \%)$, however, with higher rates than those reported in this study [21]. A study from Iran that included 19 MBL-producing $P$. aeruginosa reported that $31.5 \%$ of their isolates carried the bla $_{\mathrm{VIM}}$ gene and $10.5 \%$

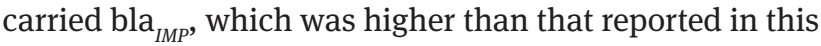
study [22]. In contrast to our study, a study from Egypt reported that none of their $P$. aeruginosa was positive for the bla ${ }_{\mathrm{VIM}}$ gene [20]. In the present study, 9.4\% of our isolates were positive for the bla ${ }_{N D M-1}$ gene, which is lower than that reported from India (27\%) [31]. A study from Bahrain reported a lower rate of bla $_{N D M-1}(2.5 \%)$ than that reported in this study [32]. In the present study, the bla ${ }_{I M P-1}$ gene was significantly higher among the isolates tested $(p=0.023)$. The $\mathrm{bla}_{I M P-1}$ gene along with bla $_{I M P-2}$ was the most common combination found in this study. There was no significant difference in the presence of MBL genes with that of hospital settings, including ICU/in-ward patients, patients under ventilator and burnt wound patients $(p>0.05)$. Fourteen (9.4\%) isolates did not amplify any of the genes tested, and these isolates could possibly carry other resistant gene variations that were not tested in this study. A study to test more resistant gene variations could possibly reveal the resistant genes associated with these isolates. The present study suggests that with the predominance of the bla $\mathrm{IMP}-1_{\mathrm{I}}$ gene, $\mathrm{bla}_{\mathrm{VIM}}$ is also increasing in our region. In addition, the high rate of the $b \mathrm{l}_{N D M-1}$ gene signifies its spread and emphasizes the need for continuous monitoring for better patient management and infection control.

\section{Conclusions}

In conclusion, the relatively high resistance of $P$. aeruginosa toward imipenem and meropenem and the high prevalence of bla $_{I M P-1}$ and $\mathrm{bla}_{\text {VIM-2 }}$ set an alarm on the increasing, perhaps the increased, carbapenem resistance. In addition to routine antibiotic susceptibility testings, our results emphasize the importance of both the phenotypic and genotypic MBL detection methods in routine practice for early detection of carbapenem resistance and to prevent further dissemination of this resistant pathogen.

Author contributions: All authors have accepted responsibility for the entire content of this manuscript and approved its submission.

Research funding: None declared.

Employment or leadership: None declared.

Honorarium: None declared.

Competing interests: Authors state no conflict of interest. Informed consent: Informed consent was obtained from all individuals included in this study.

Ethical approval: Research involving human subjects complied with all relevant national regulations, institutional policies and is in accordance with the tenets of the Helsinki Declaration (as revised in 2013), and has been approved by the authors' institutional human Ethics Committee (037-2017).

\section{References}

1. Rosenthal VD, Bijie H, Maki DG, Mehta Y, Apisarnthanarak A, Medeiros EA, et al. International Nosocomial Infection Control Consortium (INICC) report, data summary of 36 countries, for 2004-2009. Am J Infect Control 2012;40:396-407.

2. Mohanty S, Maurya V, Gaind R, Deb M. Phenotypic characterization and colistin susceptibilities of carbapenem-resistant of Pseudomonas aeruginosa and Acinetobacter spp. J Infect Dev Ctries 2013;7:880-7.

3. Strateva T, Yordanov D. Pseudomonas aeruginosa - a phenomenon of bacterial resistance. J Med Microbiol 2009;58(Pt 9):1133-48.

4. Queenan AM, Bush K. Carbapenemases: the versatile beta-lactamases. Clin Microbiol Rev 2007;20:440-58.

5. WHO publishes list of bacteria for which new antibiotics are urgently needed [https://www.who.int/en/news-room/detail/2702-2017-who-publishes-list-of-bacteria-for-which-new-antibiotics-are-urgently-needed]. 
6. Bush K, Jacoby GA. Updated functional classification of betalactamases. Antimicrob Agents Chemother 2010;54:969-76.

7. Meletis G, Bagkeri M. Pseudomonas aeruginosa: multidrug-resistance development and treatment options. In: Basak C, editor. Infection Control. Rijeka, Croatia: IntechOpen, 2013: 33-56.

8. Zhao WH, Hu ZQ. Epidemiology and genetics of VIM-type metallo-beta-lactamases in Gram-negative bacilli. Future Microbiol 2011;6:317-33.

9. Hong DJ, Bae IK, Jang IH, Jeong SH, Kang HK, Lee K. Epidemiology and characteristics of metallo-beta-lactamaseproducing Pseudomonas aeruginosa. Infect Chemother 2015;47:81-97.

10. Ramakrishnan K, Rajagopalan S, Nair S, Kenchappa P, Chandrakesan SD. Molecular characterization of metallo beta-lactamase producing multidrug resistant Pseudomonas aeruginosa from various clinical samples. Indian J Pathol Microbiol 2014;57:579-82.

11. Cornaglia G, Giamarellou H, Rossolini GM. Metallo-betalactamases: a last frontier for beta-lactams? Lancet Infect Dis 2011;11:381-93.

12. Zavascki AP, Barth AL, Goncalves AL, Moro AL, Fernandes JF, Martins AF, et al. The influence of metallo-beta-lactamase production on mortality in nosocomial Pseudomonas aeruginosa infections. J Nat Sci Biol Med 2006;58:387-92.

13. CLSI. M100-S25 performance standards for antimicrobial. Clinical and laboratory standards institute 950 west valley road, suite 2500 Wayne, PA 19087, USA, 2015.

14. Behera B, Mathur P, Das A, Kapil A, Sharma V. An evaluation of four different phenotypic techniques for detection of metallo-beta-lactamase producing Pseudomonas aeruginosa. Indian J Med Microbiol 2008;26:233-7.

15. Azimi A, Peymani A, Pour PK. Phenotypic and molecular detection of metallo-beta-lactamase-producing Pseudomonas aeruginosa isolates from patients with burns in Tehran, Iran. Rev Soc Bras Med Trop 2018;51:610-5.

16. Shibata N, Doi Y, Yamane K, Yagi T, Kurokawa H, Shibayama $\mathrm{K}$, et al. PCR typing of genetic determinants for metallo-betalactamases and integrases carried by gram-negative bacteria isolated in Japan, with focus on the class 3 integron. J Clin Microbiol 2003;41:5407-13.

17. Jovcic B, Lepsanovic Z, Suljagic V, Rackov G, Begovic J, Topisirovic L, et al. Emergence of NDM-1 metallo-beta-lactamase in Pseudomonas aeruginosa clinical isolates from Serbia. Antimicrob Agents Chemother 2011;55:3929-31.

18. Chen Y, Zhou Z, Jiang Y, Yu Y. Emergence of NDM-1-producing Acinetobacter baumannii in China. J Antimicrob Chemother 2011;66:1255-9.

19. Michalopoulos A, Falagas ME, Karatza DC, Alexandropoulou P, Papadakis E, Gregorakos L, et al. Epidemiologic, clinical characteristics, and risk factors for adverse outcome in multiresistant gram-negative primary bacteremia of critically ill patients. Am J Infect Control 2011;39:396-400.

20. Abaza AF, El Shazly SA, Selim HS, Aly GS. Metallo-beta-lactamase producing Pseudomonas aeruginosa in a Healthcare Setting in Alexandria, Egypt. Pol J Microbiol 2017;66:297-308.

21. Li Y, Zhang X, Wang C, Hu Y, Niu X, Pei D, et al. Characterization by phenotypic and genotypic methods of metallo-betalactamase-producing Pseudomonas aeruginosa isolated from patients with cystic fibrosis. Mol Med Rep 2015;11:494-8.

22. Kazeminezhad B, Bostanmanesh Rad A, Gharib A, Zahedifard S. blaVIM and blaIMP genes detection in isolates of carbapenem resistant $P$. aeruginosa of hospitalized patients in two hospitals in Iran. Iran J Pathol 2017;12:392-6.

23. Hu F, Guo Y, Yang Y, Zheng Y, Wu S, Jiang X, et al. Resistance reported from China antimicrobial surveillance network (CHINET) in 2018. Eur J Clin Microbiol Infect Dis 2019;38:2275-81.

24. Xu J, Duan X, Wu H, Zhou Q. Surveillance and correlation of antimicrobial usage and resistance of Pseudomonas aeruginosa. a hospital population-based study. PLoS One 2013;8:e78604.

25. Kakeya H, Yamada K, Nakaie K, Takizawa E, Okada Y, Fujita A, et al. [A comparison of susceptibility of Pseudomonas aeruginosa clinical isolates to carbapenem antibiotics in our hospital]. Jpn J Antibiot 2014;67:241-8.

26. Lee HS, Loh YX, Lee JJ, Liu CS, Chu C. Antimicrobial consumption and resistance in five Gram-negative bacterial species in a hospital from 2003 to 2011. J Microbiol Immunol Infect 2015;48:647-54.

27. Chung DR, Song JH, Kim SH, Thamlikitkul V, Huang SG, Wang H, et al. High prevalence of multidrug-resistant nonfermenters in hospital-acquired pneumonia in Asia. Am J Respir Crit Care Med 2011;184:1409-17.

28. Matos EC, Matos HJ, Conceicao ML, Rodrigues YC, Carneiro IC, Lima KV. Clinical and microbiological features of infections caused by Pseudomonas aeruginosa in patients hospitalized in intensive care units. Rev Soc Bras Med Trop 2016;49:305-11.

29. Ghasemian A, Salimian Rizi K, Rajabi Vardanjani H, Nojoomi F. Prevalence of clinically isolated metallo-beta-lactamase-producing Pseudomonas aeruginosa, coding genes, and possible risk factors in Iran. Iran J Pathol 2018;13:1-9.

30. Jabalameli F, Taki E, Emaneini M, Beigverdi R. Prevalence of metallo-beta-lactamase-encoding genes among carbapenemresistant Pseudomonas aeruginosa strains isolated from burn patients in Iran. Rev Soc Bras Med Trop 2018;51:270-6.

31. Mohanam L, Menon T. Coexistence of metallo-beta-lactamaseencoding genes in Pseudomonas aeruginosa. Indian J Med Res 2017;146(Supplement):S46-52.

32. Joji RM, Al-Rashed N, Saeed NK, Bindayna KM. Detection of VIM and NDM-1 metallo-beta-lactamase genes in carbapenem-resistant Pseudomonas aeruginosa clinical strains in Bahrain. J Lab Physicians 2019;11:138-43. 